Publ. RIMS, Kyoto Univ.

15 (1979), 455-468

\title{
Un Exemple dans le Problème de Cauchy pour les Equations Faiblement Hyperboliques
}

Par

Sigeo TARAMA*

\section{$\S 1$. Introduction}

On considère le problème de Cauchy pour l'opérateur différentiel dont le polynôme caractéristique est $\tau^{2}-\exp \left(-2 t^{-n}\right) \xi^{2}$ et étudie la condition pour que ce problème soit bien posé.

D’après Oleinik-Radkevich [6], Mizohata [2], le problème de Cauchy

$$
\left\{\begin{array}{l}
\left(\frac{\partial}{\partial t}\right)^{2} u-t^{2 k}\left(\frac{\partial}{\partial x}\right)^{2} u+t^{s} \frac{\partial}{\partial x} u=0 \text { dans }[0, T] \times R^{1} \\
\left(\frac{\partial}{\partial t}\right)^{i} u(0, x)=u_{i}(x), i=0,1
\end{array}\right.
$$

où $k$ et $s$ sont des nombres entiers $k \geq 1, s \geq 0$, est bien posé, si et seulement si $s \geq k-1$.

Quant aux opérateurs faiblement hyperboliques contenant l'exemple mentionné ci-dessus, on sait la condition suffisante pour que le problème de Cauchy soit bien posé (Oleinik [5], Menikoff [1], Ohya [4]). Mais comme la question est assez compliquée, on ne sait pas encore si ce type de conditions est nécessaire ou non pour des cas généraux. Donc on va donner et examiner un autre exemple pour éclaircir cette situation.

Soit $L$ l'opérateur différentiel

$$
L=\left(\frac{\partial}{\partial t}\right)^{2}-A^{2} \exp \left(-2 t^{-n}\right)\left(\frac{\partial}{\partial x}\right)^{2}+B t^{-n-1-l} \exp \left(-t^{-n}\right) \frac{\partial}{\partial x}
$$

où $A, B, n$ et $l$ sont des constantes: $A>0, n>0, l$ réel, $B \neq 0$ complexe. Pour cet opérateur $L$ on considére le problème de Cauchy

Communiqué par S. Matsuura, le 13 avril, 1977.

* Section de Mathématique et Physique appliquées Faculté des Sciences techniques, Université de Kyoto 
(C)

$$
\left\{\begin{array}{l}
L u=0,[0, T] \times R^{1} \\
\left(\frac{\partial}{\partial t}\right)^{i} u(0, x)=u_{i}(x), i=0,1 .
\end{array}\right.
$$

D’après Nersesjan [3], lorsque $l \leq 0$, le problème (C) est bien posé. Dans cet article on propose la condition nécessaire et suffisante pour que ce problème soit bien posé.

Dans le $\mathrm{n}^{\circ} 2$ on précise la notion "bien posé" et énonce le résultat. Dans les $\mathrm{n}^{\text {os }} 3$ et 4 on montre les propositions nécessaires pour démontrer le théorème dont la démonstration sera donnée dans le $n^{\circ} 4$.

L'auteur remercie Prof. Ohya pour ses conseils et encouragements.

\section{§ 2. Définitions et Résultat}

Soit $T$ un nombre positif, et soit $\Omega$ un des ensembles $[0, T] \times R^{1}$, ou $R^{1}$. On désigne par $C^{\infty}(\Omega)$ l'espace des fonctions à valeurs complexes indéfiniment dérivables dans $\Omega$, et par $C_{0}^{\infty}(\Omega)$ l'espace des fonctions à support compact et appartenant à $C^{\infty}(\Omega)$.

Définition. On dit que le problème (C) est bien posé, si pour les données $u_{1}$ et $u_{2}$ appartenant à $C^{\infty}\left(R^{1}\right)$, il $y$ a une et une seule solution du problème (C) dans $C^{\infty}\left([0, T] \times R^{1}\right)$.

Pour le problème (C) on obtient le

Théorème. Au cas où $\operatorname{Re} B=0$ (resp. $\operatorname{Re} B \neq 0$ ) le problème (C) est bien posé si et seulement si $l \leq n$ (resp. $l \leq 0)$.

Soit ${ }^{t} L$ l'opérateur formellement adjoint de $L$, c'est-ì-dire:

(2.2) ${ }^{t} L u=\left(\frac{\partial}{\partial t}\right)^{2} u-A^{2} \exp \left(-2 t^{-n}\right)\left(\frac{\partial}{\partial x}\right)^{2} u-B t^{-n-1-l} \exp \left(-t^{-n}\right) \frac{\partial}{\partial x} u$.

On considère le problème de Cauchy pour le passé

$\left(\mathrm{C}^{*}\right)$

$$
\left\{\begin{array}{l}
{ }^{t} L u=f \quad \text { dans } \quad[0, T] \times R^{1} \\
\left(\frac{\partial}{\partial t}\right)^{i} u(T, x)=0, i=0,1 .
\end{array}\right.
$$

Sur la bande $[\delta, T] \times R^{1}$, quel que soit $0<\delta<T,{ }^{t} L$ est strictement 
hyperbolique et les racines caractéristiques sont $\pm A \exp \left(-t^{-n}\right)$. Donc on sait que le problème $\left(\mathrm{C}^{*}\right)$ considéré sur la bande $\left.] 0, T\right] \times R^{1}$ est bien posé et

$\left.\left.\operatorname{supp} u \subset \bigcup_{\left(t_{0}, x_{0}\right) \in \operatorname{supp} f}\{(t, x) \in] 0, T\right] \times R^{1}|| x-x_{0} \mid \leq A \exp \left(-T^{-n}\right)\left(t_{0}-t\right)\right\}$.

Cela montre l'unicité d'une solution et l'existence de domaine d'influence comme ci-dessus pour le problème $\left(\mathrm{C}^{*}\right)$ sur la bande $[0, T] \times R^{1}$. Par conséquent s'il existe une solution dans $C^{\infty}\left([0, T] \times R^{1}\right)$ du problème (C*) avec le second membre $f \in C_{0}^{\infty}\left([0, T] \times R^{1}\right)$, on voit que le problème $\left(\mathrm{C}^{*}\right)$ sur la bande $[0, T] \times R^{n}$ est bien posé et il en résulte l'unicité d'une solution et l'existence de domaine d'influence pour le problème $(C)$. Donc pour démontrer que le problème (C) est bien posé, il suffit de montrer l'existence d'une solution du problème (C) avec les données $u_{0}, u_{1} \in C_{0}^{\infty}\left(R^{1}\right)$ et celle du problème $\left(C^{*}\right)$ sur la bande $[0, T] \times R^{1}$ avec le second membre $f \in C_{0}^{\infty}\left([0, T] \times R^{1}\right)$.

D'autre part quand le problème $(C)$ est bien posé, par le théorème du graphe fermé, il y a un ensemble compact $K$ dans $R^{1}$ et un nombre entier $m>0$ tels que la solution de (C) vérifie l'inégalité

$$
\begin{array}{r}
\sum_{i+j \leq 1} \sup _{(t, x) \in[0,(T / 2)] \times[-1,1]}\left|\left(\frac{\partial}{\partial t}\right)^{i}\left(\frac{\partial}{\partial x}\right)^{j} u(t, x)\right| \\
\leq m \sum_{\substack{0 \leq j \leq m \\
i=0,1}} \sup _{x \in K}\left|\left(\frac{\partial}{\partial x}\right)^{j} u_{i}(x)\right|
\end{array}
$$

Pour démontrer le théorème, on considère l'opérateur $\widehat{L}$ avec un paramètre $\xi$;

$$
\widehat{L} u=\frac{d^{2}}{d t^{2}} u+A^{2} \exp \left(-2 t^{-n}\right) \xi^{2} u+i B t^{-n-l-1} \exp \left(-t^{-n}\right) \xi u
$$

et étudie le comportement d'une solution $u(t, \xi)$ de $\widehat{L} u=0$ lorsque $|\xi|$ tend vers $+\infty$. Pour faire cela on se sert de la méthode utilesée par Mizohata [2].

$\S 3$. On montre les propositions concernant le comportement d'une solution $u(t, \xi)$ de $\widehat{L} u=0$. Dans cette section les lettres " $A, B, n$ et $l$ " signifient les constantes dans la définition de l'opérateur $L$ de (1.2) ( $\widehat{L}$ de $(2.4)$ ). Par le changement linéaire de la variable $x$ on peut supposer que 
$|B|<\frac{A^{2}}{2}$. Dans la suite on le suppose.

Proposition 1. Si $l>n$, alors il $y$ a des nombres positifs $M$ et $N$ tels que la solution de

$$
\left\{\begin{array}{l}
\widehat{L}_{u}=0 \\
\frac{d}{d t} u\left(t_{1}, \xi\right)=u\left(t_{1}, \xi\right)=1
\end{array}\right.
$$

satisfasse les inégalités suivantes

$$
\begin{aligned}
& |u(0, \xi)|^{2}+\left|\frac{d}{d t} u(0, \xi)\right|^{2} \leq C_{1} \\
& \left|u\left(t_{2}, \xi\right)\right|^{2}+\left|\frac{d}{d t} u\left(t_{2}, \xi\right)\right|^{2} \geq C_{2}(1+|\xi|)^{\alpha(\log |\xi|)^{\beta}}
\end{aligned}
$$

pour tout $\xi$ tel que $|\xi|>M$ et $\operatorname{Re} i B \xi \leq 0$; où $t_{1}$ et $t_{2}$ sont des nombres positifs $0<t_{1}<t_{2}<T$ définis par

$$
t_{1}=(2 \log |\xi|)^{-1 / n}, t_{2}^{n+1+l} \exp \left(-t_{2}^{-n}\right)=\frac{1}{N|\xi|}
$$

et $C_{1}, C_{2}, \alpha$ et $\beta$ sont des constantes positives indépendantes de $\xi, t_{1}, t_{2}$.

Remarque 1. Si $n+l+1 \geq 0$, alors $t^{n+l+1} \exp \left(-t^{-n}\right)$ est strictement croissante et $t_{2}$ est défini uniquement.

Remarque 2. Quand $|\xi|>1$ et $0 \leq t \leq t_{1}$, tous les coefficients de $\hat{L}$ sont uniformément bornés.

Remarque 3. Lorsque $|\xi|$ est grand, $t_{2}$ devient plus grand que $t_{1}$, et sur l'intervalle $\left[t_{1}, t_{2}\right]$ le terme dans \{\} de $\hat{L}=\frac{d^{2}}{d t^{2}}-\left\{\frac{A^{2}}{B} t^{n+1+l}\right.$ $\left.\times \exp \left(-t^{-n}\right) i \xi-1\right\} i B t^{-n-l-1} \exp \left(-t^{-n}\right) \xi$ tend vers -1 quand $N \rightarrow \infty$.

Remarque 4. Quand $|\xi|$ est grand, $t_{2}$ se situe dans l'intervalle $\left[(\log |\xi|)^{-1 / n},\left(\frac{1}{2} \log |\xi|\right)^{-1 / n}\right]$.

Proposition 2. Si $l \leq 0$, alors la solution $u(t, \xi)$ de $\hat{L} u=0$ satisfait 
l'inégalité

$$
\begin{aligned}
& \left|u\left(t_{1}, \xi\right)\right|^{2}+\left|\frac{d}{d t} u\left(t_{1}, \xi\right)\right|^{2} \\
& \quad \leq C(1+|\xi|)^{k}\left(\left|u\left(t_{2}, \xi\right)\right|^{2}+\left|\frac{d}{d t} u\left(t_{2}, \xi\right)\right|^{2}\right)
\end{aligned}
$$

pour tous $t_{1}, t_{2}$ dans $[0, T]$, où $C$ et $k$ sont des constantes positives indépendantes de $\xi, t_{1}, t_{2}$.

Les preuves des propositions 1 et 2 sont domnées dans le $11^{\circ} 5$.

Proposition 3. Si $0<l \leq n$, alors il y' a un nombre positif $M$ tel que, soit $|\xi|>M$, pour tous $t_{1}, t_{2}$ appartenant à l'intervalle $\left[0,\left(\log |\xi|-\frac{3 n+1-l}{n} \log \log |\xi|\right)^{-1 / n}\right]$, la solution de $\hat{L} u=0$ satisfasse l'inégalité suivante

$$
\begin{aligned}
& \left|u\left(t_{1}, \xi\right)\right|^{2}+\left|\frac{d}{d t} u\left(t_{1}, \xi\right)\right|^{2} \\
& \quad \leq C(1+|\xi|)^{k}\left(\left|u\left(t_{2}, \xi\right)\right|^{2}+\left|\frac{d}{d t} u\left(t_{2}, \xi\right)\right|^{2}\right)
\end{aligned}
$$

où $C$ et $k$ sont des constantes positives indépendendantes de $\xi, t_{1}, t_{2}$.

Remarque 5. Posons $t_{\xi}=\left(\log |\xi|-\frac{3 n+1-l}{n} \log \log |\xi|\right)^{-1 / n}$. Quand $|\xi|$ est grand et $n \geq l$, alors on a

$$
\xi^{2} \exp \left(-2 t^{-n}\right) \geq|\xi| \exp \left(-t^{-n}\right) t^{-n-1-l}
$$

pour tout $t \geq t_{\xi}$; en effet, l'application $|\xi| \exp \left(-t^{-n}\right) t^{n+1+l}$ est croissante, et si l'on note que $t_{\xi}>(\log |\xi|)^{-1 / n}$ quand $|\xi|$ est assez grand, alors on a

$$
\begin{aligned}
|\xi| \exp \left(-t_{\xi}^{-n}\right) t_{\xi}^{n+1+l} & =(\log |\xi|)^{(3 n+1-t) / n} t_{\xi}^{n+1+l} \\
& \geq(\log |\xi|)^{(2 n-2 l) / n} \\
& \geq 1
\end{aligned}
$$


Preuve de la proposition 3. Ci-dessous, on désigne par $C$ et $k$ des constantes arbitraires.

Par le changement des fonctions inconnues $v_{1}=u, v_{2}=\frac{d}{d t} u-$ i $A \exp \left(-t^{-n}\right) \xi u, \widehat{L} u=0$ se transforme en

$$
\begin{aligned}
& \frac{d}{d t}\left[\begin{array}{l}
v_{1} \\
v_{2}
\end{array}\right]=\left[\begin{array}{l}
i A \exp \left(-t^{-n}\right) \xi \\
-i n A t^{-n-1} \exp \left(-t^{-n}\right) \xi-i B t^{-n-1-l} \exp \left(-t^{-n}\right) \xi
\end{array}\right. \\
& \left.-i A \exp \left(-t^{-n}\right) \xi\right]\left[\begin{array}{l}
v_{1} \\
v_{2}
\end{array}\right] \text {. }
\end{aligned}
$$

Prenons $M>0$ assez grand, tel qu'on ait, pour tout $\xi$ tel que $|\xi|>M$, $T \geq(\log |\xi|)^{-1 / n}>0$ et $\frac{1}{2} \log |\xi|>\frac{3 n+1-l}{n} \log \log |\xi|>0$. Et dans la suite on considère le cas où $|\xi|>M$.

Posons $t_{1}=(\log |\xi|)^{-1 / n}$. Par l'hypothèse $0<l \leq n$ et l'intégration par parties, on a $\int_{0}^{t_{1}} \exp \left(-t^{-n}\right) t^{-n-1-l} d t \leq C(\log |\xi|)|\xi|^{-1}$; en effet $\int_{0}^{t_{1}} \exp \left(-t^{-n}\right) t^{-n-1-l} d t=\frac{1}{n} \exp \left(-t^{-n}\right) t^{-l} \int_{0}^{t_{1}}+\left.\right|_{10} ^{t_{1}} \frac{l}{n} \exp \left(-t^{-n}\right) t^{-l-1} d t$ et $\int_{0}^{t_{1}} \exp \left(-t^{-n}\right) t^{-l-1} d t \leq C \int_{0}^{t_{1}} \exp \left(-t^{-n}\right) t^{-n-1} d t \leq C \exp \left(-t_{1}^{-n}\right) . \quad$ Par la définition de $t_{1}$, on obtient la majoration.

Pour $E(t, \xi)=\left|v_{1}\right|^{2}+\left|v_{2}\right|^{2}$, on a de (3.6) $\frac{d}{d t} E(t, \xi) \mid \leq C\left\{\exp \left(-t^{-n}\right)\right.$ $\left.\times t^{-n-1-l}|\xi|+1\right\} E(t, \xi)$, d'où on obtient, pour $0 \leq s_{1}, s_{2} \leq t_{1}$,

$$
E\left(s_{2}, \xi\right) \leq C(1+|\xi|)^{k} E\left(s_{1}, \xi\right) \text { 。 }
$$

Cela montre l'inégalité (3. 4) dans l'intervalle $\left[0, t_{1}\right]$.

Posons $t_{2}=\left(\log |\xi|-\frac{3 n+1-l}{n} \log \log |\xi|\right)^{-1 / n}$. Pour $E_{2}(t, \xi)=$ $\exp \left(-t^{-n}\right) t^{-n-1-l}|\xi|\left|v_{1}\right|^{2}+\left|v_{2}\right|^{2}$, (3.6) implique

$$
\left|\frac{d}{d t} E_{2}\right| \leq\left(C_{1} t^{-n-1}+C_{2} \exp \left(-\frac{1}{2} t^{-n}\right) t^{-(n+1+l) / 2}|\xi|^{1 / 2}\right) E_{2} .
$$

En notant que $\exp \left(-t_{2}^{-n}\right)=|\xi|^{-1}(\log |\xi|)^{(3 n+1-l) / n}$ et que $0<l \leq n$, on a par l'intégration par parties

$$
\int_{t_{1}}^{t_{2}} \exp \left(-\frac{1}{2} t^{-n}\right) t^{-(n+1+l) / 2} d t \leq C_{1}(\log |\xi|)|\xi|^{-1 / 2}
$$

Donc on déduit de (3.7)

$$
E_{2}\left(s_{2}, \xi\right) \leq C(1+|\xi|)^{k} E_{2}\left(s_{1}, \xi\right)
$$


pour tous $s_{1}, s_{2} \in\left[t_{1}, t_{2}\right]$; d'où on a l'inégalité (3. 4).

C.Q.F.D.

§4. On continue à étudier le comportement d'une solution de l'équation $\widehat{L} u=0$. On suppose encore que $\frac{A^{2}}{2}>|B|$ comme dans le $\mathrm{n}^{\circ} 3$.

Prenons $M$ assez grand pour que l'on ait, pour tout $\xi$ tel que $|\xi|>M$, $T \geq(\log |\xi|)^{-1 / n}$ et $\frac{1}{2} \log |\xi|>\frac{3 n+1-l}{n} \log \log |\xi|>0$. Alors on a la

Proposition 4. Soient $0<l \leq n$ et $\operatorname{Re} B=0$. La solution de $\hat{L} u$ $=0$ satisfait l'inégalité suivante, soit $\xi$ nombre réel tel que $|\xi|>M$, pour tous $t_{1}, t_{2} \in\left[\left(\log |\xi|-\frac{3 n+1-l}{n} \log \log |\xi|\right)^{-1 / n}, T\right]$,

(4.1) $\left|u\left(t_{1}, \xi\right)\right|^{2}+\left|\frac{d}{d t} u\left(t_{1}, \xi\right)\right|^{2} \leq C(1+|\xi|)^{k}\left(\left|u\left(t_{2}, \xi\right)\right|^{2}+\frac{d}{d t} u\left(t_{2}, \xi\right)^{\left.\right|^{2}}\right)$

où $C$ et $k$ sont des constantes positives indépendantes de $t_{1}, t_{2}, \xi$.

Preuve de la proposition 4. Soit $B=i \beta$ tel que $\beta \neq 0$ et réel. Par l'hypothèse on a $A^{2} \geq 2|\beta|$. On considère l'équation

(4.2) $\frac{d}{d t}\left[\begin{array}{l}u_{1} \\ u_{2}\end{array}\right]=\left[\begin{array}{cc}0 & 1 \\ -A^{2} \exp \left(-2 t^{-n}\right) \xi^{2}+\beta \exp \left(-t^{-n}\right) t^{-n-1-l} \xi & 0\end{array}\right]\left[\begin{array}{l}u_{1} \\ u_{2}\end{array}\right]$

Posons $t_{\xi}=\left(\log |\xi|-\frac{3 n+1-l}{n} \log \log |\xi|\right)^{-1 / n}$. D'après (3.5) de la remarque 5 , on a dans l'intervalle $\left[t_{\xi}, T\right]$

$$
-\frac{1}{2} A^{2} \exp \left(-2 t^{-n}\right) \xi^{2}+\beta \exp \left(-t^{-n}\right) t^{-n-1-l} \xi \leq 0 .
$$

Soit $\lambda$ un nombre complexe tel que

$$
\lambda^{2}=-A^{2} \exp \left(-2 t^{-n}\right) \xi^{2}+\beta \xi \exp \left(-t^{-n}\right) t^{-n-1-\iota}
$$

alors on a $\operatorname{Re} \lambda=0$ et $|\lambda|^{2} \geq \frac{1}{2} A^{2} \exp \left(-2 t^{-n}\right) \xi^{2} \geq \frac{1}{2} A^{2}$ pour tout $t$ $\in\left[t_{\xi}, T\right]$. Pour la matrice

$$
N=\left[\begin{array}{rr}
\lambda & 1 \\
\lambda & -1
\end{array}\right],
$$


on pose

$$
\left[\begin{array}{l}
v_{1} \\
v_{2}
\end{array}\right]=N\left[\begin{array}{l}
u_{1} \\
u_{2}
\end{array}\right]
$$

De (4.2) on a

$$
\frac{d}{d t}\left[\begin{array}{l}
v_{1} \\
v_{2}
\end{array}\right]=\left[\begin{array}{rr}
\lambda & 0 \\
0 & -\lambda
\end{array}\right]\left[\begin{array}{l}
v_{1} \\
v_{2}
\end{array}\right]+\frac{\lambda_{t}}{2 \lambda}\left[\begin{array}{ll}
1 & 1 \\
1 & 1
\end{array}\right]\left[\begin{array}{l}
v_{1} \\
v_{2}
\end{array}\right]
$$

D'autre part on a

$$
\left|\frac{\lambda_{t}}{\lambda}\right| \leq 2 n t^{-n-1}+(n+1+l) t^{-1} \quad \text { dans } \quad\left[t_{\xi}, T\right]
$$

en effet (4. 4) s'obtient du calcul direct de $\frac{\left(\lambda^{2}\right)_{t}}{2 \lambda^{2}}$ et des majorations $|\lambda|^{2}$ $\geq \frac{1}{2} A^{2} \exp \left(-2 t^{-n}\right) \xi^{2}$ et $\left|\beta \xi \exp \left(-t^{-n}\right) t^{-n-1-l}\right| \leq \frac{A^{2}}{2} \exp \left(-2 t^{-n}\right) \xi^{2}$, dans $\left[t_{\xi}, T\right]$.

Posons $E(t, \xi)=\left|v_{1}\right|^{2}+\left|v_{2}\right|^{2}$. En vertu de (4.3) et (4.4), on a $\left|\frac{d}{d t} E(t, \xi)\right| \leq C t^{-n-1} E(t, \xi)$. Et par la majoration

$$
\int_{t_{\xi}}^{T} t^{-n-1} d t \leq C(\log |\xi|)
$$

l'inégalité (4.1) s'ensuit de la majoration

$$
C_{1}\left(\left|u_{1}\right|^{2}+\left|u_{2}\right|^{2}\right) \leq E(t, \xi) \leq C_{2}(1+|\xi|)^{2}\left(\left|u_{1}\right|^{2}+\left|u_{2}\right|^{2}\right)
$$

pour tout $t \in\left[t_{\xi}, T\right]$.

Proposition 5. Si $0<l \leq n$ et $\operatorname{Re} B \neq 0$, alors il $y$ a des nombres positifs $N$ et $\delta$ tels que, soit $\xi$ un nombre réel tel que $|\xi|>N$, et soit $t_{\xi}=\left(\log |\xi|-\frac{3 n+1-l}{n} \log \log |\xi|\right)^{-1 / n}$, la solution $u(t, \xi)$ de

$$
\left\{\begin{array}{l}
\hat{L} u=0 \\
u\left(t_{\xi}, \xi\right)=\frac{d}{d t} u\left(t_{\xi}, \xi\right)=1
\end{array}\right.
$$

satisfasse l'inégalité, pour tout $t$ dans $\left[t_{\xi}, \delta\right]$,

(4. 5) $\quad|u(t, \xi)|^{2}+\left|\frac{d}{d t} u(t, \xi)\right|^{2} \geq C \exp \left(-\gamma t^{-n-l}\right)(1+|\xi|)^{\alpha(\log |\xi|)^{\beta}}$ 
où $C, \alpha, \beta$ et $\gamma$ sont des constantes positives indépendantes de $\xi, t$.

Preuve. Ci-dessous on prend $M$ comme dans la proposition 4 et on se limite au cas où $|\xi|>M$.

Posons $t_{\xi}=\left(\log |\xi|-\frac{3 n+1-l}{n} \log \log |\xi|\right)^{-1 / n}$. Soit $B=-\beta+i \alpha$ où $\alpha$ et $\beta$ sont réels et $\beta \neq 0$, et soit $\lambda$ un nombre complexe tel que $\lambda^{2}$ $=-A^{2} \exp \left(-2 t^{-n}\right) \xi^{2}+\alpha \exp \left(-t^{n}\right) t^{-n-1-l} \xi+i \beta t^{-1-n-l} \exp \left(-t^{-n}\right) \xi$ et que $\operatorname{Re} \lambda>0$. (3.5) de la remarque 5 dans le $\mathrm{n}^{\circ} 3$ et l'hypothèse $\frac{1}{2} A^{2}$ $>|B|$ montrent que pour tout $t \in\left[t_{\xi}, T\right]$

$$
\frac{3}{2} A^{2} \exp \left(-2 t^{-n}\right) \xi^{2} \geq|\lambda|^{2} \geq \frac{A^{2}}{2} \exp \left(-2 t^{-n}\right) \xi^{2}
$$

Et on a

$$
\operatorname{Re} \lambda \geq k t^{-n-1-l} \text { pour } t>t_{\xi}
$$

en effect soit $\lambda^{2}=r \exp (i \theta)$ où $r$ et $\theta$ sont réels. On a

$$
\operatorname{Re} \lambda=r^{1 / 2} \cos \frac{\theta}{2} \geq r^{-1 / 2} \cdot \frac{r}{2}|\sin \theta|
$$

et

$$
\begin{aligned}
& r^{1 / 2}=|\lambda| \leq \sqrt{\frac{3}{2}} A \exp \left(-t^{-n}\right)|\xi| \\
& |r \sin \theta|=\left|\operatorname{Im} \lambda^{2}\right|=|\beta| t^{-n-1-l} \exp \left(-t^{-n}\right)|\xi|
\end{aligned}
$$

d'où (4. 6).

Par la majoration comme (4.4) dans la preuve de la proposition 4, on a

$$
\left|\frac{\lambda_{t}}{\lambda}\right| \leq C t^{-n-1} \quad t_{\xi} \leq t \leq T
$$

En vertu de (4.6), (4.7) et de l'hypothèse $0<l \leq n$, on peut prendre $\delta>0$ asse $z$ petit et $N>M$ assez grand tels que l'on ait

$$
t_{\xi}<\delta \text { pour }|\xi|>N
$$

$$
\operatorname{Re} \lambda>2\left|\frac{\lambda_{t}}{\lambda}\right| \text { pour } \quad t_{\xi} \leq t \leq \delta
$$


Par le changement des fonctions inconnues

$$
\begin{aligned}
& {\left[\begin{array}{l}
v_{1} \\
v_{2}
\end{array}\right]=\left[\begin{array}{rr}
\lambda & 1 \\
\lambda & -1
\end{array}\right]\left[\begin{array}{l}
u_{1} \\
u_{2}
\end{array}\right],} \\
& \frac{d}{d t}\left[\begin{array}{l}
u_{1} \\
u_{2}
\end{array}\right]=\left[\begin{array}{cc}
0 & 1 \\
-A^{2} \exp \left(-2 t^{-n}\right) \xi^{2}-i B t^{-n-1-l} \exp \left(-t^{-n}\right) \xi & 0
\end{array}\right]\left[\begin{array}{l}
u_{1} \\
u_{2}
\end{array}\right]
\end{aligned}
$$

se transforme en

$$
\frac{d}{d t}\left[\begin{array}{l}
v_{1} \\
v_{2}
\end{array}\right]=\left[\begin{array}{rr}
\lambda & 0 \\
0 & -\lambda
\end{array}\right]\left[\begin{array}{l}
v_{1} \\
v_{2}
\end{array}\right]+\frac{\lambda_{t}}{2 \lambda}\left[\begin{array}{ll}
1 & 1 \\
1 & 1
\end{array}\right]\left[\begin{array}{l}
v_{1} \\
v_{2}
\end{array}\right]
$$

Posons $S(t, \xi)=\left|v_{1}\right|^{2}-\left|v_{2}\right|^{2}$. On a

$$
\frac{d}{d t} S(t, \xi) \geq 2 \operatorname{Re} \lambda\left(\left|v_{1}\right|^{2}+\left|v_{2}\right|^{2}\right)-2\left|\frac{\lambda_{t}}{\lambda}\right|\left(\left|v_{1}\right|^{2}+\left|v_{2}\right|^{2}\right)
$$

De (4.8) et de (4.9) on a

$$
S(t, \xi) \geq \exp \left(\int_{t_{\xi}}^{t} \operatorname{Re} \lambda d t\right) S\left(t_{\xi}, \xi\right) \quad \text { pour } \quad|\xi|>N \quad \text { et pour } t \in\left[t_{\xi}, \delta\right]
$$

d'où on obtient

$$
S(t, \xi) \geq C_{1} \exp \left(-\frac{k}{n+l} t^{-n-l}\right)(1+|\xi|)^{C_{2}(\log |\xi|)^{l / n}} S\left(t_{\xi}, \xi\right)
$$

pour $t_{\xi} \leq t \leq \delta$, tenant compte de (4.6). Et par l'hypothèse $0<l \leq n$ on a l'inégalité (4.5).

C.Q.F.D.

Démonstration du théorème. Compte tenu des propositions, on peut démontrer le théorème. En effet, au cas où $\operatorname{Re} B=0$, si $l>n$, on prend la solution $u$ de $\hat{L} u=0$ considérée dans la proposition 1 et pose

$$
v(t, x)=u(t, \xi) \exp (i x \xi) .
$$

Alors $v(t, x)$ satisfait l'équation $L v=0$; mais d'après la proposition 1 , quel que soit $m>0$, l'inégalité (2.3) n'est pas valide pour $v(t, \xi)$ quand $|\xi|$ est assez grand. Cela montre que, si $\operatorname{Re} B=0$ et $l>n$, le problème (C) n'est pas bien posé. Si $l \leq n$, par la transformation de Fourier par rapport à la variable $x$ et par les majorations obtenues dans les propositions 2 , 3 et 4 , on voit que le problème de Cauchy (C) (resp. $\left(C^{*}\right)$ ) avec les données $u_{0}, u_{1} \in C_{0}^{\infty}\left(R^{1}\right)$ (resp. avec le second membre $f \in$ 
$\left.C_{0}^{\infty}\left([0, T] \times R^{1}\right)\right)$ a une solution dans $C^{\infty}\left([0, T] \times R^{1}\right)$, et tenant compte de la remarque suivant l'énoncé du théorème dans le $\mathrm{n}^{\circ} 2$, on peut conclure que le problème de Cauchy $(C)$ est bien posé quand $\operatorname{Re} B=0$ et $l \leq n$. Ce qui achève la démonstration du théorème au cas où $\operatorname{Re} B=0$. Au cas où $\operatorname{Re} B \neq 0$, on peut raisonner de la même façon.

$\S 5$. On donne les preuves des propositions 1 et 2 . On désigne par $C, k$ des constantes arbitaires.

Prenve de la proposition 1. D'après la remarque 2 dans le $\mathrm{n}^{\circ} 3$, on a aisément l'inégalité (3.1).

Posons $v_{1}=u(t, \xi)$ et $v_{2}=\frac{d}{d t} u(t, \xi) . \quad \hat{L} u=0$ se transforme en

(5. 1) $\frac{d}{d t}\left[\begin{array}{l}v_{1} \\ v_{2}\end{array}\right]=\left[\begin{array}{cc}0 & 1 \\ -A^{2} \exp \left(-2 t^{-n}\right) \xi^{2}-i B t^{-n-1-2} \exp \left(-t^{-n}\right) \xi & 0\end{array}\right]\left[\begin{array}{l}v_{1} \\ v_{2}\end{array}\right]$

En considérant le terme

$$
\left[\begin{array}{cc}
0 & 1 \\
-i B t^{-n-1-l} \exp \left(-t^{-n}\right) \xi & 0
\end{array}\right]
$$

comme la partie principale, on va le faire diagonal.

Soit $\lambda=\sqrt{-i B \xi /|\xi|}$ tel que $\operatorname{Re} \lambda>0$ pour $\xi$ tel que $-\operatorname{Re} i B \xi \geq 0$ et soit $N$ la matrice

$$
\left[\begin{array}{lr}
|\xi|^{1 / 2} \lambda \exp \left(-\frac{1}{2} t^{-n}\right) t^{-(n+1+l) / 2} & 1 \\
|\xi|^{1 / 2} \lambda \exp \left(-\frac{1}{2} t^{-n}\right) t^{-(n+1+l) / 2} & -1
\end{array}\right] .
$$

Par le changement des fonctions inconnues

$$
\left[\begin{array}{l}
w_{1} \\
w_{2}
\end{array}\right]=N\left[\begin{array}{l}
v_{1} \\
v_{2}
\end{array}\right]
$$

(5.1) se met sous

(5. 2) $\frac{d}{d t}\left[\begin{array}{l}w_{1} \\ w_{2}\end{array}\right]=\left[\begin{array}{rr}\lambda & 0 \\ 0 & -\lambda\end{array}\right] t^{-(n+1+l) / 2} \exp \left(-\frac{1}{2} t^{-n}\right)|\xi|^{1 / 2}\left[\begin{array}{c}w_{1} \\ w_{2}\end{array}\right]$ 


$$
\begin{aligned}
& +\left\{-A^{2} \frac{1}{2 \lambda} \exp \left(-t^{-n}\right)|\xi| t^{n+1+l}\left[\begin{array}{rr}
1 & 1 \\
-1 & -1
\end{array}\right] t^{-(n+1+l) / 2} \exp \left(-\frac{1}{2} t^{-n}\right)|\xi|^{1 / 2}\right. \\
& \left.+\left(\frac{n}{2} t^{-n-1}-\frac{n+1+l}{2} t^{-1}\right)\left[\begin{array}{ll}
1 / 2 & 1 / 2 \\
1 / 2 & 1 / 2
\end{array}\right]\right\}\left[\begin{array}{c}
w_{1} \\
w_{2}
\end{array}\right] .
\end{aligned}
$$

En posant $S(t, \xi)=\left|w_{1}\right|^{2}-\left|w_{2}\right|^{2}$, on obtient de (5.2)

$$
\begin{aligned}
\frac{d}{d t} S(t, \xi) \geq & 2 \operatorname{Re} \lambda \cdot t^{-(n+1+l) / 2} \exp \left(-\frac{1}{2} t^{-n}\right)|\xi|^{1 / 2}\left(\left|w_{1}\right|^{2}+\left|w_{2}\right|^{2}\right) \\
- & \frac{2 A^{2}}{\mid \lambda !} \exp \left(-t^{-n}\right)|\xi| t^{n+1+l} t^{-(n+1+l) / 2} \\
& \times \exp \left(-\frac{1}{2} t^{-n}\right)|\xi|^{1 / 2}\left(\left|w_{1}\right|^{2}+\left|w_{2}\right|^{2}\right) \\
+ & \left(\frac{n}{2} t^{-n-1}-\frac{n+1+l}{2} t^{-1}\right)\left(\left|w_{1}\right|^{2}-\left|w_{2}\right|^{2}\right) .
\end{aligned}
$$

Ici on prend $N$ assez grand, en définissant $t_{2}$ par $t_{2}^{n+1+l} \exp \left(-t_{2}^{-n}\right)$ $=\frac{1}{N|\xi|}$, pour qu'on ait $|\xi| \frac{2 A^{2}}{|\lambda| \operatorname{Re} \lambda} \exp \left(-t^{-n}\right) t^{n+1+l} \leq 1$ pour $0<t<t_{2}$ (Voir la remarque 1 dans le $\mathrm{n}^{\circ} 3$ ). Alors si $0<t<t_{2}$, on a

$$
\begin{aligned}
\frac{d}{d t} S(t, \xi) \geq & (\operatorname{Re} \lambda) t^{-(n+1+) l / 2} \exp \left(-\frac{1}{2} t^{-n}\right)|\xi|^{1 / 2} S \\
& +\left(\frac{n}{2} t^{-n-1}-\frac{n+1+l}{2} t^{-1}\right) S
\end{aligned}
$$

d'où on a

$$
\text { (5.3) } \begin{aligned}
S\left(t_{2}, \xi\right) \geq & \exp \left\{\operatorname{Re} \lambda \int_{t_{1}}^{t_{2}} t^{-(n+1+l) / 2} \exp \left(-\frac{1}{2 t^{n}}\right)|\xi|^{1 / 2} d t\right\} \\
& \times t_{2}^{-(n+1+l) / 2} \exp \left(-\frac{1}{2} t_{2}^{-n}\right) t_{1}^{(n+1+l) / 2} \exp \left(\frac{1}{2} t_{1}^{-n}\right) S\left(t_{1}, \xi\right) .
\end{aligned}
$$

Soit $M$ un nombre positif tel que, pour $|\xi|>M$, on ait

$$
(\log |\xi|)^{-1 / n} \leq t_{2} \leq\left(\frac{1}{2} \log |\xi|\right)^{-1 / n}
$$

et

$$
\exp \left(-\frac{1}{2} t_{2}^{-n}\right) t_{2}^{(n-l) / 2}>2 \exp \left(-\frac{1}{2} t_{1}^{-n}\right) t_{1}^{(n-l) / 2}
$$


vu la remarque 4 dans le $n^{\circ} 3$. Donc on a pour $\xi$ tel que $|\xi|>M$

$$
\int_{t_{1}}^{t_{2}} t^{-(n+1+l) / 2} \exp \left(-\frac{1}{2} t^{-n}\right)|\xi|^{1 / 2} d t \geq C(\log |\xi|)^{1+(l-n) / n}
$$

en effet

$$
\begin{aligned}
& \int_{t_{1}}^{t_{2}} t^{-(n+1+l) / 2} \exp \left(-\frac{1}{2} t^{-n}\right)|\xi|^{1 / 2} d t \\
& \geq t_{1}^{1 / 2} \int_{t_{1}}^{t_{2}} t^{-(n+l) / 2-1} \exp \left(-\frac{1}{2} t^{-n}\right)|\xi|^{1 / 2} d t \\
& \geq t_{1}^{1 / 2}\left\{\left.\frac{2}{n} \exp \left(-\frac{1}{2} t^{-n}\right) t^{-(l-n) / 2}|\xi|^{1 / 2}\right|_{t_{1}} ^{t_{2}}\right. \\
& \left.\quad+\frac{l-n}{n} \int_{t_{1}}^{t_{2}} \exp \left(-\frac{1}{2} t^{-n}\right) t^{-(l-n) / 2-1}|\xi|^{1 / 2} d t\right\} .
\end{aligned}
$$

Puisque $l>n$ et $|\xi|>M$

$$
\geq t_{1}^{1 / 2} \frac{1}{n} \exp \left(-\frac{1}{2} t_{2}^{-n}\right) t_{2}^{-(l-n) / 2} \mid \xi^{1 / 2}
$$

et par les définition de $t_{1}$ et de $t_{2}$ on a l'inégalité (5.4). D'autre part

$$
S(t, \xi)=4 \operatorname{Re}\left(\lambda \exp \left(-\frac{1}{2} t^{-n}\right) t^{-(n+1+l) / 2}|\xi|^{1 / 2} v_{1} \bar{v}_{2}\right)
$$

En notant que $\left.v_{1} \bar{v}_{2}\right|_{t=t_{1}}=1$, en vertu de (5.3), (5.4) et de (5.5) on obtient l'inégalité (3.2).

Preuve de la Proposition 2. Quand $|\xi|<N$ quel que soit $N$ un nombre positif, on a aisément l'inégalité (3.3) puisque les coefficients sont uniformément bornés.

Dans l'intervalle $\left[0, t_{1}\right]$ où $t_{1}=(\log |\xi|)^{-1 / n}$, l'inégalité (3. 3) découle de la preuve de la proposition 3 .

Par le changement des fonctions inconnues $u_{1}=\xi u, u_{2}=\frac{d}{d t} u, \hat{L} u$ $=0$ devient

(5. 6) $\frac{d}{d t}\left[\begin{array}{l}u_{1} \\ u_{2}\end{array}\right]=\left[\begin{array}{cc}0 & , \xi \\ -A^{2} \exp \left(-2 t^{-n}\right) \xi-i B t^{-n-1-l} \exp \left(-t^{-n}\right), & 0\end{array}\right]\left[\begin{array}{l}u_{1} \\ u_{2}\end{array}\right]$.

En considérant le terme 
$\left[\begin{array}{ccc}0 & , \xi \\ -A^{2} \exp \left(-2 t^{-n}\right) \xi, & 0\end{array}\right]$ comme la partie principale, on va le faire diagonal.

Posons

$$
\left[\begin{array}{l}
v_{1} \\
v_{2}
\end{array}\right]=N\left[\begin{array}{l}
u_{1} \\
u_{2}
\end{array}\right] \text { où } N=\left[\begin{array}{lr}
i A \exp \left(-t^{-n}\right), & 1 \\
i A \exp \left(-t^{-n}\right), & -1
\end{array}\right] .
$$

L'équation (5.6) se transforme en

$$
\begin{aligned}
\frac{d}{d t}\left[\begin{array}{l}
v_{1} \\
v_{2}
\end{array}\right]= & {\left[\begin{array}{rr}
1 & 0 \\
0 & -1
\end{array}\right] i A \exp \left(-t^{-n}\right) \xi\left[\begin{array}{l}
v_{1} \\
v_{2}
\end{array}\right] } \\
& +\frac{n}{2} t^{-n-1}\left[\begin{array}{ll}
1 & 1 \\
1 & 1
\end{array}\right]\left[\begin{array}{l}
v_{1} \\
v_{2}
\end{array}\right]-\frac{B}{2 A} t^{-n-1-l}\left[\begin{array}{rr}
1 & 1 \\
-1 & -1
\end{array}\right]\left[\begin{array}{l}
v_{1} \\
v_{2}
\end{array}\right] .
\end{aligned}
$$

Par l'hypothèse $l \leq 0$, on a

$$
\left|\frac{d}{d t} E(t, \xi)\right| \leq C t^{-1-n} E(t, \xi) \quad \text { pour } \quad t_{1} \leq t \leq T \text { où } E(t, \xi)=\left|v_{1}\right|^{2}+\left|v_{2}\right|^{2} \text {. }
$$

Notant que

$$
\begin{aligned}
& \int_{t_{1}}^{\infty} t^{-n-1} d t=\frac{1}{n} \log |\xi| \text { et que, pour } t_{1} \leq t \leq T \\
& C_{1}(1+|\xi|)^{-2}\left(\left|u_{1}\right|^{2}+\left|u_{2}\right|^{2}\right) \leq E(t, \xi) \leq C_{2}\left(\left|u_{1}\right|^{2}+\left|u_{2}\right|^{2}\right),
\end{aligned}
$$

on a la majoration à montrer.

\section{Bibliographie}

[1] Menikoff, A., The Cauchy problem for weakly hyperbolic equations, Amer. J. Math., 97 (1975), 548-558.

[2] Mizohata, S., Une remarque sur le problème de Cauchy hyperbolique bien posé (non publié).

[3] Nersesjan, A. B., The Cauchy problem for degenerating hyperbolic equations of second order, Soviet Math. Dokl., 7 (1966), 278-281.

Izv. Akad. Nauk Arm. SSR, 3 (1968), 79-100 ('’article détaillé).

[4] Ohya, Y., Le problème de Cauchy à caractéristiques multiples-méthode directe pour obtenir la condition (généralisée) de E. E. Levi-, C. R. Acad. Sc. Paris, 282 (1976), 1433-1436.

[5] Oleinik, O. A., On the Cauchy problem for weakly hyperbolic equations, Comm. Pure Appl. Math., 23 (1970), 569-586.

[6] Oleinik, O. A. and Radkevich, E. V., Second order equations with nonnegative characteristic form, Amer. Math. Soc., Providence, Rhode Island and Plenum Press, New York, 1973. 